\title{
Trait-based approaches to global change ecology: from description to prediction
}

\author{
Stephanie Green ${ }^{1}$, Cole Brookson ${ }^{1}$, Natasha Hardy ${ }^{1}$, and Larry Crowder ${ }^{2}$ \\ ${ }^{1}$ University of Alberta \\ ${ }^{2}$ Stanford University
}

July 28, 2020

\begin{abstract}
As global change forces species' ranges and abundances into novel configurations, traits-based approaches could allow predictions of community re-assembly. We present a quantitative review of traits-based research globally to (1) evaluate the extent to which this approach has been applied, and (2) evaluate moving from description and to prediction. We highlight the application of traits-based frameworks to describe ecological patterns; terrestrial plant morphology comprises $>30 \%$ of the literature alone. But fewer than $3 \%$ of studies predict ecological effects of global change, mostly in the past five years. While organism size is the most common trait, we identified 2,430 other morphological, physiological, behavioural, and life history traits that mediate environmental filters of species' ranges across ecosystems and taxonomy. Global change studies forecast range shifts from a few physiological or life history traits. Though uncommon, spatially-explicit models constructed from correlated multivariate trait assemblages (or 'syndromes') offer the best chance of predicting shifts under global change scenarios. Moving the field towards trait-based prediction requires (1) matching the scale of trait measurement to the ecological processes, (2) increasing the resolution of environmental gradients along which traits are measured, (3) moving from single to multivariate traits, and (4) accounting for intraspecific trait variation.
\end{abstract}

\section{Introduction}

Identifying general principles that govern the distribution and abundance of species across Earth's ecosystems is a fundamental pursuit in ecology. Over the past three decades, 'trait-based' ecology - focused on the role that measurable characteristics of organisms play in mediating geographic distribution and abundance - has emerged as a major conceptual lens through which to describe general processes that might drive patterns of biodiversity across the biosphere (Hansen and Urban1992; McGill et al. 2006; Mindel et al. 2016). Traitsbased theory stemmed from descriptive correlations between the frequency of phenotypic traits hypothesized to affect individual and population level success across taxa and environmental contexts (e.g. Grime 1988; Diaz and Cabido 1997). But the ultimate potential of traits-based approaches might allow scientists to predict ecological outcomes in new contexts (i.e. functional traits; McGill et al. 2006).

The need for predictive trait-based approaches is increasingly urgent due to mounting evidence that stresses like climate change, biological invasion, and over-exploitation are having profound effects on ecosystems and the socioeconomic benefits they provide. While ecological communities are inherently dynamic, with species membership and relative abundance varying over time and space (Cottenie 2005, Dornelas et al. 2014), unprecedented levels of anthropogenic stress are now driving species range and density changes that far exceed historical levels (Chapin et al. 2000, IUCN 2008). Effects are proving to be unequal across species (Zavaleta et al. 2009, Sunday et al. 2012, Bates et al. 2014), so ecological communities are essentially being pulled apart, and reassembled with new member combinations (Hobbs et al. 2009, García Molinos et al. 2015). What will future species assemblages look like, and how will they function, in the face of these major disturbances? Identifying species' characteristics that recur across unrelated taxa and confer information 
about species performance under a range of environmental and biotic interactions offers the potential to predict ecological dynamics as novel ecosystem configurations form under global change. For example, as abiotic conditions defining the fundamental niche shift under climate change, biotic traits that confer species' dispersal and survival abilities help define the shape of realized niches they are likely to occupy (McGill 2006; Early and Sax 2011; Estrada et al. 2018).

Here we present a quantitative review of trait-based biodiversity research and its application to global change ecology to address the following questions: (1) Which traits offer promising insights into the outcomes of environmental and biotic filtering across ecosystem types and taxonomic groups? (2) To what extent (and in what contexts) are traits-based insights being applied to predict the ecological outcomes of global change? (3) What are the potential barriers to predicting the outcome of environmental and biotic interactions using traits? (4) What research techniques and conceptual frameworks can help us move beyond describing ecological patterns and towards prediction? Our review is framed from the perspective that species' behavioural, morphological, physiological, and life history attributes mediate scale-dependent environmental and biotic filters on distribution and abundance across land and seascapes (Figure 1). Species' characteristics that confer success under abiotic environmental conditions such as temperature, light, acidity, moisture (in terrestrial systems) and dissolved oxygen (in aquatic systems) provide the coarsest filter on species distribution (i.e. Filter 1 in Figure 1; the boundaries of the fundamental niche). Traits that influence the outcome of biotic interactions within local environments further mediate species persistence and co-existence (i.e. including trophic interactions; Filter 3 in Figure 1). A host of traits that confer information on species' dispersal ability mediate feedbacks between the effects of abiotic and biotic interactions on species' range and relative abundances (i.e. dispersal-limited controls on redistribution; Filter 2 in Figure 1). Knowledge of the role that traits play in filtering across these scales facilitates prediction about species persistence and relative abundance in new contexts such as altered environmental conditions (e.g. climate-mediated shifts in thermal regimes; Figure $1 \mathrm{~A}$ vs B).

\section{Analytical framework}

To synthesize current work on trait-based approaches for predicting ecological dynamics under global change, we conducted a systematic review of published literature on the topic via structured keyword searches in online internationally recognized databases (Supplementary Materials 1). We classified the resulting studies according to eight attributes related to the insights traits provided into ecological change: (1) the type(s) of traits examined in the study (life history, morphological, behavioural, physiological) (2) the specific organismal traits that were quantified in the study, (3) the scale of environmental filtering on which the research focused (abiotic matching, dispersal, or biotic interactions [which was further refined into trophic interactions], as in Figure 1), (4) the ecosystem and (5) taxonomic focus, (6) the research methodology used (experimental or observational study of differences among groups, reviews and metanalyses, or theoretical modelling), (7) whether a driver of global change was examined, and if so, the type (e.g. climate change, biological invasion, habitat degradation), and (8) crucially, whether or not the study included predictions for ecological outcomes beyond the data set for which the analysis was initially constructed. In addition to summarizing trends in trait-based research over time and in these eight domains, we visualized similarities and differences between the assemblages of traits used to investigate ecological processes across studies representing different levels of these factors through non-metric multidimensional scaling (nMDS) plots, generated from dissimilarity matrices of the multivariate trait data and grouped by levels of the factors 3-8 above (Supplementary Materials 2a). Finally, we identified trait types contributing to differences between domains of trait-based research by constructing rank abundance curves and evaluating multivariate generalized linear models of the traits used in the literature, again grouped by factors 3-8 above (Supplementary Materials 2b).

\section{Current trends in trait-based ecology}

In total, we identified 822 studies relevant to traits-based ecology from $>200$ journals published as early as 1978. Over $50 \%$ of the studies came from just 18 journals. The number of studies applying traits-based approaches to describe ecological pattern and process has grown exponentially over the past forty years, with a marked increase in the rate at which traits-based ecological studies have been published in the last decade 
(Figure 2). Most studies (59\%) focused on the role of traits at a single level of environmental filtering, while only $3.5 \%$ of studies examined traits in the context of more than two levels of filtering. One study, UsseglioPolatera (2000 - Freshwater Biology ), examined traits in the context of deriving environmental filtering from coarsest (i.e. abiotic environment matching) to finest (i.e. trophic interactions) filters. In total, 435 studies examined more than one type of trait (i.e. physiological, morphological, behavioural, or life history), 176 considered more than two trait types, and only $26(<0.5 \%)$ studies considered traits that represented all four categories simultaneously.

Most of the research to date focuses on relating variation in morphological and life history features of vascular plants to abiotic environmental filtering processes within terrestrial ecosystems using observational techniques (Figures 3 and 4; 34\% of all papers focused on plants, 30\% on plant morphology); this result that is perhaps unsurprising given that traits-based research originated in plant ecology community (McGill et al. 2006). In contrast, traits-based investigations within marine and freshwater systems comprises just $30 \%$ of the studies combined, with a focus on observational studies of traits in the context of abiotic environmental filtering processes, primarily for fishes (Figures 3 and 4).

Trait-based research outside of plant-based research has overwhelmingly focused on size as the characteristic of interest (Figure 5, Table S3, Figs S3-S14). In fact, we identified 131 different metrics of size used across studies, representing a range of length, mass, or volume measures -including vegetative height and cone length (plants), snout-ventral length or instar size (animals), and biovolume (cells). Body size is the most common trait measured in studies of animal taxa, and also the most variable in terms of its definition and measurement, with metrics ranging from wet or dry mass, to inference from the length of the organism (involving multiple methods of measurement depending on the study and taxa under consideration). In contrast, specific leaf area (SLA; measured as leaf area per dry mass) is the most common trait used within plant trait research and is estimated via a single s standardized method across studies and plant taxa (Evans \& Hughes 1961; Spence et al. 1973). In fact, SLA is the most common single trait examined in the studies reviewed here (127 occurrences over the 822 studies), with measures of body size a close second at 108 occurrences.

Beyond size, a plethora of morphological, behavioural, life history, and physiological features have been applied to trait-based biodiversity research (Figure 5). In total, we identified 2,561 unique traits within the literature we reviewed. While only $5 \%$ of these (131) represented aspects of organism size, size-based traits were used within 405 studies $(49 \%)$ and each of these 131 traits were used by 3.7 separate papers on average, while non-size traits were each used only 1.7 times on average across the 822 studies. Accounting for variation in trait names (i.e. 'time to maturity' vs. 'age at maturity'), and instances where a similar trait was assessed via multiple metrics (e.g. 'trophic guild' could be measured as a categorical variable with levels 'resource', 'primary consumer', 'secondary consumer', or 'tertiary consumer', while in a separate study 'trophic group' could be measured in levels of 'primary producer', 'herbivore', 'omnivore', or 'carnivore', both relating to 'trophic role') allowed us to attribute traits that confer information about the same process into conceptual groupings, revealing 196 'secondary' trait classifications (Supplementary Material; Table S3). Of these, $14 \%$ are used in a single study while $34 \%$ are used in more than 10 studies. Of the 2,561 unique traits, morphological traits (Figure $5 ; \mathrm{n}=1,163$ ) included aspects of organisms' physical form (e.g. body size and shape, or the presence and form of dentition or spines) and biochemical composition (e.g. nitrogen or carbon content). Key behavioural traits (Figure $5, \mathrm{n}=626$ ) include aspects of organisms' activity (e.g. movement rates or nocturnality) and habitat use (e.g. vertical habitat position within forest canopies or water columns, range size or edge position). Life history traits (Figure $5 ; \mathrm{n}=585$ ) describe growth, abundance, survival, and reproduction (including reproductive mode, timing, and frequency), while physiological traits (Figure 5; $\mathrm{n}=187$ traits) conferred information about organisms' environmental habitat requirements (e.g. moisture or temperature tolerances), and resource acquisition (e.g. photosynthetic rate).

The diversity and identity of traits applied to ecological research depend on the environmental filter under investigation (Figure 1; Figure 5A-D, Figure S7\&S8) and the ecosystem of interest (Figure 5I-K, Figure S9\&S10). Interestingly, relatively narrower assemblages of traits are used in plant compared with animal 
research (Fig 6A,Fig S1A\&S2A), with several potential explanations: (i) plant ecology has focused on traits for longer, and thus may have made relatively more progress in distilling a specific set of traits that represent key processes (versus animal trait research, which still needs to be distilled in this way), (ii) traits that represent key processes have more standardized measurement methods than traits for other taxonomic groups (i.e. plant size measured as SLA vs the variety of animal body size measurements), and (iii) differences in size of the trait space between taxonomic groups may simply reflect differences between sessile/mobile organisms; Compared with mobile taxa, sessile plants and fungi (represented in 'other'; Figure 6A, Figure S12) have a relatively more narrow set of strategies for resource acquisition, defense, dispersal, and reproduction. In addition, perhaps also due to the longer history of traits approaches to plant ecology, research linking plant functional traits to their hypothesized ecological function is somewhat more developed, conferring a more complete understanding of the ecological processes that can be inferred from specific traits.

We also find that relatively narrow assemblages of traits have been applied in experiments, meta-analyses, reviews, and theoretical work compared with observational research (Figure 6B), the latter being the majority of studies in this review, and the likely starting place for first identifying and linking traits to important aspects of species distribution and interactions. Likewise, a relatively narrow assemblage of traits are applied within multi-ecosystem studies (i.e. those where findings are relevant broadly across ecosystem types; Figure $6 \mathrm{C})$. However, the multi-ecosystem research reviewed here primarily represent theoretical models, which apply traits that can be estimated universally across taxa and systems such as body size and shape, and feeding mode, potentially explaining why the assemblage of traits applied in this body of work is more homogenous compared with marine, freshwater, or terrestrial focused-work (Figure 6C, Figure S14).

\section{Traits-based global change predictions}

Of the 822 studies we reviewed, a small portion (23\%) focused on applying traits in the context of global change, and even fewer (3\%) applied traits to generate predictions about patterns of diversity, abundance, or distribution beyond the data used for the initial analysis. Global change drivers, in order of decreasing frequency within the studies we evaluated, included habitat degradation $(8 \%)$, climate change $(6 \%)$, biological invasion (5\%), multiple/non-specified drivers (3\%), and exploitation (1\%). Predictive studies emerged primarily within the last 10 years $(82 \%)$; half of all predictive studies were published in the last five years (Figure $2 \mathrm{~F}$ ). More than $30 \%$ of predictive studies focused on applying plant morphological traits to predict the outcomes of abiotic environmental filtering in terrestrial ecosystems. Crucially, studies that generated trait-based predictions of global change (the main focus of this review) represent fewer than $3 \%$ (22) of all studies. Of these, more than half (12) focus on ecological prediction in a climate change context, three on biological invasions, and a single study each on the consequences of habitat degradation and exploitation. Five studies used traits to predict the outcomes of multiple global change drivers (two marine [Jacob et al. 2011, Eklof et al. 2015]; three terrestrial [Cardillo et al. 2004; Dury et al. 2018; Knott et al. 2019]).

The narrowest subset of journal articles (and subsequently, traits) corresponded to studies that were both predictive and investigated global change impacts to ecosystems $\left(n_{\text {studies }}=22\right)$. Accordingly, the assemblage of traits used by papers that assessed drivers of global change and performed predictive ecological modelling were highly nested within the broader suite of traits used across descriptive and non-global change studies, and thus differed statistically with traits used more broadly (Figures 6E/F, Figures S1E/F, Figure S2E/F). Habitat associations and life history were the most important suites of traits in studies assessing climate change impacts, as well as undertaking predictive analyses, while morphological traits were most important to studies that investigated habitat degradation and biological invasions (Figure S3\&S4, Tables S4-8). While size is also the most common single trait type in predictive studies, in general physiological traits related to resource acquisition and requirements, such as thermal tolerance, and life history traits are more often applied within predictive studies compared with descriptive trait-based work (Figures S5\&S6, Tables S8-11).

More closely examining predictive global change studies identified in this review highlights a range of methodological approaches that require data inputs at varied spatio-temporal scales, and therefore resulting in predictions at a range of resolutions (Figure 7). Each method has strengths and weaknesses; trait-based experiments offer opportunities to generate and test fine-scale predictions about response to global change 
drivers, yet insights gained through experimentation are most relevant under the set of conditions under which the study takes place (e.g. Eckloff et al. 2015; eelgrass communities under climate change and grazer loss; Figure 7). The results of experiments and environmental correlations can be synthesized via metaanalyses to generalize effect sizes for trait types that recur across taxa and ecosystem type (e.g. Cattano et al. 2018's synthesis of acidification effects on marine fishes; Figure 7). Process models offer an opportunity to examine the effect of more complex interactions on ecological phenomena under global change; however, insights gained from this approach may not be at a resolution needed for conservation and management decision-making (e.g. Jacob et al. 2011's trait-based polar sea food web model; Figure 7). Traits-based distribution models - the most common approach to global change predictions identified by our review - have generally been applied to forecasting range and abundances under future abiotic (climate) conditions (e.g. Fordham et al. 2012's and Whitney et al. 2017's distribution models of trees and freshwater fish, respectively; Figure 7). Spatial projections generated from distribution models can be intuitively applied to place-based biodiversity conservation and natural resource management, but generally omit biotic interactions and feedbacks that further refine species' ranges and abundances across the landscape (Figure 1). Spatially explicit process models offer a means to generate range and abundance projections that account for multiple environmental filtering processes simultaneously (e.g. Edmunds et al. 2014's spatial process model of coral survival and growth under competition and climate warming scenarios; Figure 7). However, this approach has not yet been widely applied to global change prediction, perhaps due to the complexity and scale of the required trait data inputs.

\section{Challenges and opportunities for global change prediction}

While our review confirms growing interest in and application of traits-based frameworks to ecological patterns, applications have primarily been descriptive - highlighting a persistent gap in application to ecological prediction, and in particular, predicting dynamics under global change. In synthesizing existing work, at least four key themes emerged in terms of guiding future work and overcoming barriers to trait-based predictions of global change outcomes: (1) matching the scale of trait measurement to the ecological processes disrupted by global change, (2) increasing the resolution of environmental and ecological gradients along which traits are measured, (3) moving beyond body size and toward multivariate trait assemblages to describe and predict ecological processes, (4) accounting for intraspecific trait variation in predicted responses to global change.

\section{Matching the scale of trait measurement to the ecological processes disrupted by global change}

Ultimately, trait-based analyses of ecological variance strive for generality in explaining ecological processes, for example size-based relationships in predator-prey interactions (Rice et al. 1993) or thermo-tolerance in species responses to environmental gradients of change (e.g. using naturally occurring climate resilient corals to construct bleaching-resistant nurseries; Morikawa and Palumbi 2019). However, relevance and specificity drive the need to down-scale trait-based approaches to the system and questions, while maintaining scalable analytical frameworks. The resolution at which traits are measured and associated with the constituents of biological communities (i.e. by individual, life stage, population, or species) determines the level of inference that can be achieved in evaluating their role in mediating outcomes across environmental and ecological contexts. We suggest that the scale at which traits should be measured depends on 1) the ecological scale of the research question (individual, population, community, or ecosystem), 2) the focus on ecological patterns or process, 3) the extent to which intra- and inter-specific variation is hypothesized to play an important role in mediating the focal environmental filtering process in question, and 4) the analytical method used to generate inference.

Trait variation can be measured for a community of organisms or at the species-level, as well as at subpopulation to individual level. It must be clear whether intra-specific variation in trait values within species or groups of taxa that share measurable traits is the required currency for predictive modeling. But species' trait information is typically available to analysts at either broad (species- or metapopulation-) scales or very specific geographic scales, with a rate-limiting middle ground of information on spatially and temporally explicit subpopulations. Where the consequences of species interactions are the focus of investigation, and food web traits are conserved at a species or population level, trait-based linkages between species groups may 
be most appropriately coded at that level (i.e., one value per species or population). Trait variation can be measured at a subpopulation to individual level, where intra-specific variation in trait values within species or groups of taxa that share measurable traits is the required currency for a model. For example, across large longitudinal or latitudinal gradients of change that span multiple species' subpopulations. However, traits of interest were typically represented using a single value/level per species in the predictive global change studies identified here (Figure 7), regardless of study type, ecosystem, or taxonomic focus.

\section{Increasing the resolution of global change gradients along which traits are measured}

Species traits are often measured along major environmental gradients (i.e. elevation or latitude), with measurements focused on capturing intraspecific variation (Chalmandrier et al. 2017), interspecific variation (Balasubramaniam \& Rottenberry, 2016), or both (Classen et al. 2017). Such gradients usually encompass a broad range of environmental factors that can be static or dynamic across space and time (i.e. water temperature, acidity, soil quality, wind, etc.). Constructing trait-based predictions requires ecologists to identify at what scale specific traits metrics change in response to particular aspects of the gradient of interest, yet trait variation is often not explicitly linked to measures of important aspect of the gradient at the same resolution (e.g. environmental data collected at the region or site level, while trait data collected at the individual level). Designing field data collection with trait and environmental data sets gathered at equal resolutions allows ecologists to quantify the scale at which variation in both response and explanatory variables matters for the environmental filtering process(s) under investigation (i.e. Figure 1). Advanced multivariate techniques (e.g. fourth corner an RQL analysis) can be used to parse out relationships between traits and different aspects of environmental gradients, as well as the spatial and temporal scale at which these relationships hold, subsequently allowing for the identification of potential ecological mechanisms driving trait and phylogenetic patterns across land and seascapes (Anderson et al. 2019). Without explicit links between directional trait variation and the aspect(s) of the environment driving that change, ecologists may continue to miss likely causal relationships that are useful for prediction.

\section{Moving beyond body size in trait-based ecological prediction}

Body size scales with key processes across all stages of environmental filtering (e.g. desiccation tolerance, metabolism, prey consumption rates, movement; Brown et al. 2004), so it is not surprising that measures of organism size are applied so frequently in trait-based ecology. While the inclusion of body size, particularly in descriptions of food webs, has been of great benefit to describing ecological structure and function (Woodward et al. 2005; Brose et al. 2006; Jacob et al. 2011), this single trait explains a limited amount of variation in terms of trait-driven biological and ecological processes. Moreover, other morphological and physiological traits are also key mediators of the influence of size, such as body shape and metabolic approach to temperature regulation, respectively. Within size classes, variation in key behavioural and life history traits can influence establishment and persistence within ecological communities; for example, reproductive behaviours are often incorporated into trait-based analyses that model species' and populations' dispersal capabilities in changing and novel ecosystems (Estrada et al. 2018). The inclusion of traits other than body size can increase our understanding of current ecological community structure/function (Gravel et al. 2016), but perhaps more importantly, our ability to provide important insights for prediction of future ecological states arising from ongoing global change. However, the role of many behavioural, life history, physiological, and morphological traits (other than size) identified in this review - ranging from fecundity, to jaw strength, to flight initiation distance - are mediated by organism size, illustrating the all importance of incorporating a range of correlated traits into predictive analyses.

\section{Measuring multivariate trait 'syndromes' to explain ecological outcomes}

Organisms' responses to their environment are governed by complex suites of correlated traits that confer important about performance under specific sets of environmental filters (Figure 1). Strong correlation among trait types that can recur across unrelated species - trait 'syndromes' or 'typologies' - underpin trait-based community assembly theory for plants (Grime 1988) and behavioural syndromes in animals (Sih et al. 2004). Yet single trait-type studies make up roughly half of the research we reviewed (387 papers) compared with 
multi-trait (i.e. 3 or more traits) studies (176 papers, 21\%), and we estimate that the true ratio of single vs. multi-trait studies to be more significantly skewed towards single-trait studies. Our review is likely conservative in assessing their prevalence because an unknown number of studies have likely been excluded from this review because they do not self-identify as trait-based investigations of ecological processes, and rather simply identify the particular trait investigated in relation to species distributions or abundances. In considering single traits at a time, as a function of a species' or ecological community's response to gradients of change, we risk overlooking the combined effect that a range of traits may have in explaining those responses to change (e.g. multivariate traits for restoration design to resist invasion; Funk et al. 2008, and plant trait typologies along ecological gradients; Diaz et al. 2004). While there has been a trend towards identifying a subset of traits that are strong indicators for ecosystem processes (e.g. Hausner et al. 2003), increasingly sophisticated statistical tools and computing power exist to deal with the greater model complexity that comes with multi-trait based analyses of ecological relationships. For example, multi-matrix modeling solutions enable the simultaneous assessment of relationships between species abundances and/or distribution data, environmental gradients of change and the role that traits play in mediating changes in biodiversity and assemblages in the face of environmental change (Dray et al. 2014, Wang et al. 2012, Brown et al. 2014). Comparatively simple techniques available in standard multivariate statistical tool kits and packages include parametric and non-parametric techniques for variable reduction and synthesis of trait combinations. Traditionally, techniques such as principal component analysis, non-metric dimensional scaling and clustering are most commonly used for identifying trait groupings and trait typologies, while systematically reducing variables included in models, and these remain powerful techniques fit for that purpose (Legendre \& Rogers 1972, Legendre \& Legendre 1998).

\section{Accounting for intraspecific trait variation in predicted responses to global change}

In our review, only 38 papers specifically reference investigating the intraspecific trait variation in their system, while interspecific variation dominated in most of the papers either explicitly or implicitly. In practice, the acquisition of high-resolution trait information measured for individuals within populations is labour intensive and often specific to a temporal, spatial, and ecological context (e.g., lipid content or energy density of prey species). Aggregate values of this information, when available, can be taken at a species level and used to model broader patterns in the responses to environmental or ecological variables of population-tospecies. The majority of large-scale trait-based modelling occurs at the population to species resolution (e.g. Spencer et al. 2019). But species do vary in the magnitude of intra- versus inter-specific variation in their traits (i.e. many species vary greatly in traits across ontogeny). Individuals within a population may possess traits that confer advantages for dispersal to or persistence within changing ecosystems (Muñoz et al. 2016, Archambault et al. 2018). Coarse resolution trait-based modelling may therefore overlook nuanced ecological processes when intraspecific variation is ignored; therein lies a trade-off between trait-to-system relevance and high data collection effort, compared to reduced information availability and low trait-to-system specificity. The choice ultimately comes down to the need for understanding system-specific processes versus trait-based synthetic products that can provide answers to practitioners and managers now but that may contain intraspecific inaccuracies. Issues with aggregating information at a population-to-species level need to be explicitly acknowledged, where for example traits are known to vary within species and at the scale of the environmental gradients examined. Best practice in trait-based analyses of ecological variance should strive for the selection of appropriate trait resolution (i.e. binary, categorical, continuous) and the scale of trait measurements (i.e., spatial/temporal scales as well as scales of intraspecific variation). Our review highlights the need for international efforts to aggregate and make accessible trait information (ranging from individual to specieslevel metrics) via platforms for knowledge sharing and reporting. Prominent examples include FishBase (for fish, Froese and Pauly 2019), SeaLifeBase (for marine invertebrates, Palomares \& Pauly 2019), TRY (for plants, Kattge et al. 2011). But many studies report undertaking significant additional curation of the information acquired from these databases. Manual curation, handling, cleaning, and archiving of trait-based data is labour intensive and costly, ideally requiring consultation with taxonomic experts, and represents a significant barrier to elaborating trait-based approaches. Online repositories that facilitate collation of regional, ecosystem-specific, or taxon-specific trait data collections could help to address some of these 
barriers, however such efforts sustained baseline funding to maintain data archives, address user issues, and continually evolve the repository and products to address emerging needs (see Gallagher et al. 2020).

\section{Re-examining the past to inform future trait-based predictions}

Ecology in the Anthropocene is characterized by the rise of cumulative effects on ecosystems, and thus there is an urgent need to synthesize current trajectories of ecological change, predict future ecological outcomes in relation to multiple drivers of change, and importantly, account for the naturally large number of components affected and effecting change. The persistence or loss of species in novel Anthropocene ecosystems will depend on several factors that may be predicted using traits: (i) species' potential responses to environmental forcing (dispersal, establishment, persistence), (ii) the capacity of species to affect community dynamics (i.e. interactions strengths), and (iii) the combined effect of multiple anthropogenic forces on organisms' interactions with the environment and one another (i.e. either additive, antagonistic, synergistic), and (iv) the type and duration of stressors (e.g. Hillebrand and Kunze 2020). Further applications of trait-based approaches to modelling and predicting ecological change are needed, as well as validations of such models. Ample testing grounds exist ideally where ecological processes are reasonably well understood and where the impacts of environmental change can be investigated in relation to that prior understanding of ecosystem form and function. For example, numerous examples of community reassembly and varying degrees of ecosystem modification are documented in the literature, particularly on trophic cascades and ecosystem engineers. These examples have helped to shape our understanding of the processes and factors involved in generating ecological outcomes of change; but hindsight also supplies much of what we know of the role of focal species' traits in driving ecological outcomes.

Using the growing body of existing trait data, ecologists are positioned to build and test trait-based predictions through hindcasting ecological outcomes in systems that continue to face rapid community reassembly. Transition zone ecosystems - for example, regions at the boundaries between tropical and temperate coastal ocean reef ecosystems in Australia, Japan, the Eastern Pacific and Western Atlantic (see Vergés et al. 2014), where prominent coastal warming and ecological mixing zones persist - are areas where suites of species are readily being redistributed due to environmental forcing. These boundary systems provide excellent opportunities to construct and test trait-based hypotheses of rapid ecological change. For example, the 'tropicalization' of temperate reef systems is occurring due to multiple and interacting environmental drivers of change (notably climate change, overfishing and the protection of ecosystems through marine park designation) afford researchers expansive opportunities to propose and test hypotheses across gradients in natural experimental setting (Text Box 1; Figure 8). Studies of key filtering traits such as thermal tolerance, larval starvation resistance, and predator avoidance strategies for many marine species in these systems have explained shifts in distribution and abundances of range expanders (Text Box 1; Figure 8). Additionally, the warming of tropical systems will likely lead to transitions to new ecological states not observed in living human time scales. These systems represent a need for generalisable, and trait-based predictive tools to forecast ecological outcomes beyond recent ecological states. We suggest that focusing research effort in this field on incorporating suites of traits into spatially-explicit process models of transition-zone ecosystems are likely to yield the most fruitful tests and validations of trait-based global change predictions.

\section{Acknowledgements}

This research was supported by Lenfest Ocean Program Grant to SJG and LBC and a Sloan Research Fellowship to SJG. We are grateful to participants in the 2019 Traitspace workshop at Chicheley Hall in Buckinghamshire, UK for feedback and discussion that has greatly enriched and improved this study.

Text Box 1: Re-examining the past to advance trait-based predictions for the future: Trait-based drivers of ecosystem re-assembly following over-exploitation and climate change

The tropicalisation of temperate reefs in coastal southeastern Australia as a result of ongoing global change represents a prominent example of abiotic and biotic traits facilitating species' establishment in novel habitats, resulting in local community phase-shifts (Figure 7). Since 1910, average ocean warming of $>1 @ C$ and a strengthening boundary current in the region have enabled poleward colonization of numerous tropical 
and sub-tropical 'vagrants', as thermal barriers to their dispersal have been eroded (Poloczanska et al. 2007, Figueira \& Booth 2010; Figueira et al. 2009, Verges et al. 2014). While many species have successfully dispersed to cooler latitudes, not all become established and fewer still go on to affect the form and function of novel ecosystems. However, the range expansion of an ecosystem engineer - the diadematid sea urchin Centrostephanus rodgersii - from tropical reefs off New South Wales (NSW) to temperate reefs off Tasmania has had major ecological repercussions, threatening kelp forest ecosystems in this region already affected by ocean warming (Johnson et al. 2005, Ling et al. 2009a).

Several abiotic, dispersal, and trophic traits likely simultaneously contributed to the successful range and abundance expansion of $C$. rodgersii under changing environmental conditions. Namely, the species possesses high dispersal potential in the larval phase (Andrew \& Byrne 2001; Johnson et al. 2005), being morphologically adapted for long distance travel in the plankton, resilient to low food quality and quantity, and with long pelagic larval duration (> 100 days; Emlet et al. 2002, Soars et al. 2009). In addition, C. rodgersii is a moderately fast growing sea urchin, likely reaching a critical size for predator avoidance within $4-5$ years even in novel higher-latitude ecosystems, they are nocturnally active and hide in complex reef habitat by day (Ling et al. 2009b, Andrew \& Byrne 2001).

While the removal of thermal barriers combined with dispersal-related life history traits facilitated initial range expansion by $C$. rodgersii, these changes also coincided with the prior growth overfishing of a key bottom-dwelling predator capable of foraging on temperate reef invertebrates including urchins - the spiny

lobsterJasus edwarsii . Both a reduction in abundances and body sizes (which influences their amount of crushing force on prey) of these predators may have effectively reduced predation pressure on colonizing sea urchins on Tasmanian reefs to levels that facilitated urchin establishment and population growth. Combined with changing abiotic conditions, predator loss from the system likely resulted in over-grazing of kelp beds, and loss of kelp-dominated ecosystems in this region (Barrett et al. 2009, Ling et al. 2009b).

\section{References}

Anderson, M. J., de Valpine, P., Punnett, A., \& Miller, A. E. (2019). A pathway for multivariate analysis of ecological communities using copulas. Ecology and Evolution, 9(6), 3276-3294.

Andrew NL (1993) Spatial heterogeneity, sea urchin grazing and habitat structure on reefs in temperate Australia. Ecology 74: 292-302

Andrew NL (1994) Survival of kelp adjacent to areas grazed by sea urchins in New South Wales, Australia. Aust J Ecol 16: 353-362

Andrew NL, Byrne M (2001) The ecology of Centrostephanus rodgersii. In: Edible Sea Urchins: Biology and Ecology (ed. Lawrence JM), pp. 149-160. Elsevier Science, Amsterdam, the Netherlands.

Archambault, J. M., Cope, W. G., \& Kwak, T. J. (2018). Chasing a changing climate: Reproductive and dispersal traits predict how sessile species respond to global warming. Diversity and Distributions, 24(7), 880-891.

Balasubramaniam, P., \& Rotenberry, J. T. (2016). Elevation and latitude interact to drive life-history variation in precocial birds: a comparative analysis using galliformes. Journal of Animal Ecology, 85(6), 1528-1539.

Barrett NS, Buxton CD, Edgar G. (2009) Changes in invertebrate and macroalgal populations in Tasmanian marine reserves in the decade following protection. J Exp Mar Biol Ecol 370.

Bates, A. E., G. T. Pecl, S. Frusher, A. J. Hobday, T. Wernberg, D. A. Smale, J. M. Sunday, N. A. Hill, N. K. Dulvy, R. K. Colwell, N. J. Holbrook, E. A. Fulton, D. Slawinski, M. Feng, G. J. Edgar, B. T. Radford, P. A. Thompson, and R. A. Watson. 2014. Defining and observing stages of climate-mediated range shifts in marine systems. Global Environmental Change 26:27-38. 
Brose, U., Jonsson, T., Berlow, E. L., Warren, P., Banasek-Richter, C., Bersier, L. F., .. \& Cushing, L. (2006). Consumer-resource body-size relationships in natural food webs. Ecology, 87(10), 2411-2417.

Brown, J. H., Gillooly, J. F., Allen, A. P., Savage, V. M., \& West, G. B. (2004). Toward a metabolic theory of ecology. Ecology, 85(7), 1771-1789.

Brown, A. M., Warton, D. I., Andrew, N. R., Binns, M., Cassis, G., \& Gibb, H. (2014). The fourth-corner solution-using predictive models to understand how species traits interact with the environment. Methods in Ecology and Evolution, 5(4), 344-352.

Cardillo, M., A. Purvis, W. Sechrest, J.L. Gittleman, J. Bielby, G.M. 2004. Human population density and extinction risk in the world's carnivores. PLoS Biolgy, 2(7), e197.

Cattano, C., Claudet, J., Domenici, P., \& Milazzo, M. (2018). Living in a high CO2 world: a global metaanalysis shows multiple trait-mediated fish responses to ocean acidification. Ecological Monographs, 88(3), 320-335.

Chalmandrier, L., Münkemüller, T., Colace, M. P., Renaud, J., Aubert, S., Carlson, B. Z., .. \& Lavergne, S. (2017). Spatial scale and intraspecific trait variability mediate assembly rules in alpine grasslands. Journal of Ecology, 105(1), 277-287.

Chapin, S. F., E. S. Zavaleta, V. T. Eviner, R. L. Naylor, P. M. Vitousek, H. L. Reynolds, D. U. Hooper, S. Lavorel, O. E. Sala, S. E. Hobbie, M. C. Mack, and S. Diaz. 2000. Consequences of changing biodiversity. Nature 405:234.

Classen, A., Steffan-Dewenter, I., Kindeketa, W. J., \& Peters, M. K. (2017). Integrating intraspecific variation in community ecology unifies theories on body size shifts along climatic gradients. Functional Ecology, 31(3), 768-777.

Cottenie, K. 2005. Integrating environmental and spatial processes in ecological community dynamics. Ecology Letters 8:1175-1182.

Dornelas, M., N. J. Gotelli, B. McGill, H. Shimadzu, F. Moyes, C. Sievers, and A. E. Magurran. 2014. Assemblage Time Series Reveal Biodiversity Change but Not Systematic Loss. Science 344:296-299.

Dray, S., Choler, P., Doledec, S., Peres-Neto, P. R., Thuiller, W., Pavoine, S., \& ter Braak, C. J. (2014). Combining the fourth-corner and the RLQ methods for assessing trait responses to environmental variation. Ecology, 95(1), 14-21.

Dury, M., Mertens, L., Fayolle, A., Verbeeck, H., Hambuckers, A., \& Francois, L. (2018). Refining species traits in a dynamic vegetation model to project the impacts of climate change on tropical trees in Central Africa. Forests, 9(11), 722 .

Early, R., \& Sax, D. F. (2011). Analysis of climate paths reveals potential limitations on species range shifts. Ecology letters, 14(11), 1125-1133.

Edmunds, P. J., Adjeroud, M., Baskett, M. L., Baums, I. B., Budd, A. F., Carpenter, R. C., .. \& Han, X. (2014). Persistence and change in community composition of reef corals through present, past, and future climates. PLoS One, 9(10), e107525.

Eklof, J. S., Havenhand, J. N., Alsterberg, C., \& Gamfeldt, L. (2015). Community-level effects of rapid experimental warming and consumer loss outweigh effects of rapid ocean acidification. Oikos, 124(8), 10401049.

Emlet RB, Young CM, George SB (2002) Phylum Echinoder-mata: Echinoidea. In: Young CM, Rice ME, Sewell MA(eds) An atlas of marine invertebrate larvae. Academic Press, London, p 531-551 
Estrada, A., Morales-Castilla, I., Meireles, C., Caplat, P., \& Early, R. (2018). Equipped to cope with climate change: traits associated with range filling across European taxa. Ecography, 41(5), 770-781.

Evans, G. C., \& Hughes, A. P. (1961). Plant growth and the aerial environment: I. Effect of artificial shading on Impatiens parviflora. New Phytologist, 60(2), 150-180.

Figueira, W. F., Biro, P., Booth, D. J., \& Valenzuela, V. C. (2009). Performance of tropical fish recruiting to temperate habitats: role of ambient temperature and implications of climate change. Marine Ecology Progress Series, 384, 231-239.

Figueira, W. F., \& Booth, D. J. (2010). Increasing ocean temperatures allow tropical fishes to survive overwinter in temperate waters. Global Change Biology, 16(2), 506-516.

Fordham, D. A., Resit Akcakaya, H., Araujo, M. B., Elith, J., Keith, D. A., Pearson, R., .. \& Tozer, M. (2012). Plant extinction risk under climate change: are forecast range shifts alone a good indicator of species vulnerability to global warming?. Global Change Biology, 18(4), 1357-1371.

Froese, R. and D. Pauly. Editors. (2019.) FishBase. World Wide Web electronic publication.www.fishbase.org, version (12/2019).

Funk, J. L., Cleland, E. E., Suding, K. N., \& Zavaleta, E. S. (2008). Restoration through reassembly: plant traits and invasion resistance. Trends in ecology \& evolution, 23(12), 695-703.

Gallagher, R. V., Falster, D. S., Maitner, B. S., Salguero-Gomez, R., Vandvik, V., Pearse, W. D.,...\& Ankenbrand, M. J. (2020). Open Science principles for accelerating trait-based science across the Tree of Life. Nature ecology \& evolution, 4(3), 294-303.

Garcia Molinos, J., B. S. Halpern, D. S. Schoeman, C. J. Brown, W. Kiessling, P. J. Moore, J. M. Pandolfi, E. S. Poloczanska, A. J. Richardson, and M. T. Burrows. 2015. Climate velocity and the future global redistribution of marine biodiversity. Nature Clim. Change advance online publication.

Gravel, D., Albouy, C., \& Thuiller, W. (2016). The meaning of functional trait composition of food webs for ecosystem functioning. Philosophical Transactions of the Royal Society B: Biological Sciences, 371(1694), 20150268.

Grime, J. P. (1988). The CSR model of primary plant strategies - origins, implications and tests. In Plant evolutionary biology (pp. 371-393). Springer, Dordrecht.

Hansen, A. J., and Urban, D. L. (1992). Avian response to landscape pattern: the role of species' life histories. Landscape Ecology, 7(3), 163-180.

Hausner, V. H., Yoccoz, N. G., \& Ims, R. A. (2003). Selecting indicator traits for monitoring land use impacts: birds in northern coastal birch forests. Ecological Applications, 13(4), 999-1012.

Hobbs, R. J., E. Higgs, and J. A. Harris. 2009. Novel ecosystems: implications for conservation and restoration. Trends in Ecology \& Evolution 24:599-605.

Hillebrand, H. and Kunze, C. (2020), Meta-analysis on pulse disturbances reveals differences in functional and compositional recovery across ecosystems. Ecol Lett, 23: 575-585. doi:10.1111/ele.13457

Hobday, A.J., and Lough, J.M. (2011). Projected climate change in Australian marine and freshwater environments. Marine and Freshwater Research 62, 1000-1014.

IUCN. 2008. Wildlife in a changing world: An analysis of the 2008 IUCN Red List of Threatened Species. Gland, Switzerland.

Jacob, U., Thierry, A., Brose, U., Arntz, W. E., Berg, S., Brey, T., .. \& \& Petchey, O. L. (2011). The role of body size in complex food webs: A cold case. In Advances in ecological research (Vol. 45, pp. 181-223). Academic Press. 
Johnson CR, Ling SD, Ross J, Shepherd S, Miller K (2005) Establishment of the long-spined sea urchin (Centrostephanus rodgersii) in Tasmania: first assessment of potential threats to fisheries. FRDC Final Report, Project No. 2001/044. School of Zoology and Tasmanian Aquaculture and Fisheries Institute, University of Tasmania.

Kattge, J., Diaz, S., Lavorel, S., Prentice, I. C., Leadley, P., Bonisch, G., . . \& Cornelissen, J. H. C. (2011). TRY-a global database of plant traits. Global change biology, 17(9), 2905-2935. URL:https://www.trydb.org/TryWeb/Home.php

Knott, J. A., Desprez, J. M., Oswalt, C. M., \& Fei, S. 2019. Shifts in forest composition in the eastern United States. Forest Ecology and Management, 433, 176-183.

Lau, D. C. C., Lau, S. C. K., Qian, P. Y., and Qiu, J. W. (2009). Morphological plasticity and resource allocation in response to food limitation and hyposalinity in a sea urchin. Journal of Shellfish Research 28, 383-388.

Legendre, P., \& Legendre, L. (1998). Numerical ecology: developments in environmental modelling. Developments in Environmental Modelling, 20.

Legendre, P., \& Rogers, D. J. (1972). Characters and clustering in taxonomy: a synthesis of two taximetric procedures. Taxon, 21(5-6), 567-606

Ling, S. D., Johnson, C. R., Frusher, S., \& King, C. K. (2008). Reproductive potential of a marine ecosystem engineer at the edge of a newly expanded range. Global Change Biology, 14(4), 907-915.

Ling, S. D., Johnson, C. R., Ridgway, K., Hobday, A. J., \& Haddon, M. (2009a). Climate-driven range extension of a sea urchin: inferring future trends by analysis of recent population dynamics. Global Change Biology, 15(3), 719-731.

Ling, S.D., Johnson, C.R., Frusher, S.D., and Ridgway, K.R. (2009b). Overfishing reduces resilience of kelp beds to climate-driven catastrophic phase shift. Proceedings of the National Academy of Sciences of the United States of America 106, 22341-22345.

Ling, S. D., and Johnson, C. R. (2009). Population dynamics of an ecologically important range-extender: kelp beds versus sea urchin barrens. Marine Ecology Progress Series 374, 113-125. doi:10.3354/MEPS07729

McGill, B. J., B. J. Enquist, E. Weiher, and M. Westoby. 2006. Rebuilding community ecology from functional traits. Trends in Ecology \& Evolution 21:178-185.

Mindel, B. L., Neat, F. C., Trueman, C. N., Webb, T. J., \& Blanchard, J. L. (2016). Functional, size and taxonomic diversity of fish along a depth gradient in the deep sea. PeerJ, 4, e2387.

Munoz, D. J., Miller Hesed, K., Campbell Grant, E. H., \& Miller, D. A. (2016). Evaluating within-population variability in behavior and demography for the adaptive potential of a dispersal-limited species to climate change. Ecology and evolution , 6 (24), 8740-8755.

Morikawa, M. K., and Palumbi, S. R. (2019). Using naturally occurring climate resilient corals to construct bleaching-resistant nurseries. Proceedings of the National Academy of Sciences, 116(21), 10586-10591.

Palomares, M.L.D. and D. Pauly. Editors. (2019). SeaLifeBase. World Wide Web electronic publication.www.sealifebase.org, version (12/2019).

Pecorino, D., Lamare, M. D., \& Barker, M. F. (2012). Growth, morphometrics and size structure of the Diadematidae sea urchin Centrostephanus rodgersii in northern New Zealand. Marine and Freshwater Research, 63(7), 624-634.

Poloczanska ES, Babcock RC, Butler A et al. (2007) Climate change and Australian marine life. Oceanography and Marine Biology: An Annual Review, 45, 407-478. 
Rice, J.A., T.J. Miller, K.A. Rose, L.B. Crowder, E.A. Marschall, A.S. Trebitz, and D.L. DeAngelis. 1993. Growth rate variation and larval survival: Inferences from an individual-based size-dependent predation model. Can. J. Fish. Aquat. Sci. 50:133-142.

Ridgway KR (2007a) Long-term trend and decadal variability of the southward penetration of the East Australian Current. Geophysical Research Letters, 34, L13613, doi:10.1029/2007GL030393.

Sih, A., Bell, A., \& Johnson, J. C. (2004). Behavioral syndromes: an ecological and evolutionary overview. Trends in ecology \& evolution, 19(7), 372-378.

Soars, N. A., Prowse, T. A. A., \& Byrne, M. (2009). Overview of phenotypic plasticity in echinoid larvae,'Echinopluteus transversus' type vs. typical echinoplutei. Marine Ecology Progress Series, 383, 113-125.

Spence, D. H. N., Campbell, R. M., \& Chrystal, J. (1973). Specific leaf areas and zonation of freshwater macrophytes. The Journal of Ecology, 317-328.

Sunday, J. M., A. E. Bates, and N. K. Dulvy. 2012. Thermal tolerance and the global redistribution of animals. Nature Clim. Change 2:686-690.

Usseglio-Polatera, P., Bournaud, M., Richoux, P., \& Tachet, H. (2000). Biological and ecological traits of benthic freshwater macroinvertebrates: relationships and definition of groups with similar traits. Freshwater Biology, 43(2), 175-205.

Verges, A., Steinberg, P. D., Hay, M. E., Poore, A. G., Campbell, A. H., Ballesteros, E., .. \& Figueira, W. (2014). The tropicalization of temperate marine ecosystems: climate-mediated changes in herbivory and community phase shifts. Proceedings of the Royal Society B: Biological Sciences, 281(1789), 20140846.

Wang, Y. I., Naumann, U., Wright, S. T., \& Warton, D. I. (2012). mvabund-an R package for model-based analysis of multivariate abundance data. Methods in Ecology and Evolution, 3(3), 471-474.

Whitney, J. E., Whittier, J. B., Paukert, C. P., Olden, J. D., \& Strecker, A. L. (2017). Forecasted range shifts of arid-land fishes in response to climate change. Reviews in Fish Biology and Fisheries, 27(2), 463-479.

Woodward, G., Ebenman, B., Emmerson, M., Montoya, J. M., Olesen, J. M., Valido, A., \& Warren, P. H. (2005). Body size in ecological networks. Trends in Ecology \& Evolution, 20(7), 402-409.

Wright JT, Dworjanyn SA, Rogers CN, Steinberg PD, Williamson JE, Poore AGB (2005) Density-dependent sea urchin grazing: differential removal of species, changes in community composition and alternative community states. Mar Ecol Prog Ser 298: 143-156

Zavaleta, E., J. Pasari, J. Moore, D. Hernandez, K. B. Suttle, and C. C. Wilmers. 2009. Ecosystem responses to community disassembly. Annals of the New York Academy of Sciences 1162:311-333. 


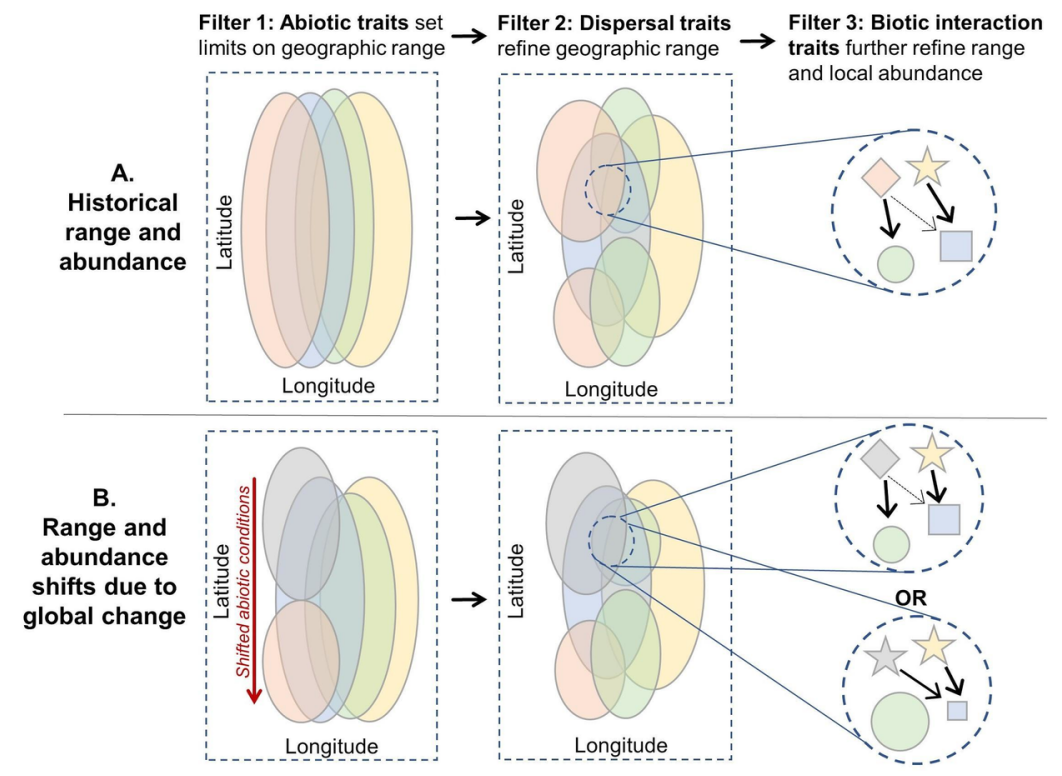

\section{Figure 1}

Figure 1. Trait-based filters of species range and abundance mediate the effects of global change on ecological communities. Ellipse $=$ species geographic range; colour $=$ species identity; shape = trait types; shape size $=$ relative abundance, arrow thickness $=$ strength of consumptive interactions within local food webs. A. Many traits (e.g. thermal tolerances) mediate species' interactions with their abiotic environment, setting limits on species' potential geographic ranges (i.e. fundamental niche space; Filter 1). Within this fundamental niche space, dispersal traits (e.g. larval duration) further restricts range (Filter 2). Finally, traits govern the strength and nature of biotic interactions (e.g. foraging mode), further refining range and modifying local abundance. B. As abiotic conditions shift, a priorknowledge of species' traits and their influence on filters 1-3 may allow us to predict changes in local species composition and abundance. For example, diamond vs star traits for the new grey species in B.

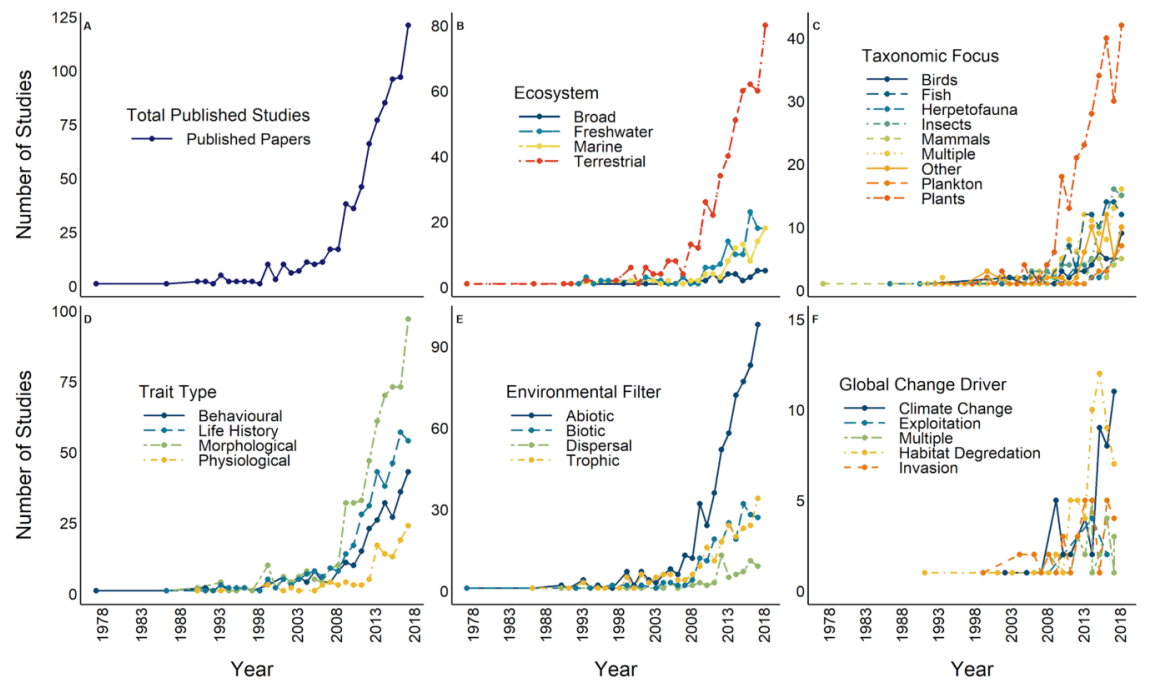




\section{Figure 2}

Figure 2 . Temporal patterns of studies published in various categories over the years covered by our review. $\mathrm{A}=$ All studies, $\mathrm{B}=$ studies by ecosystem type, $\mathrm{C}=$ studies by taxonomic focus, $\mathrm{D}=$ studies by trait type, $\mathrm{E}=$ studies by level of environmental filtering, $\mathrm{F}=$ studies by global change drivers.

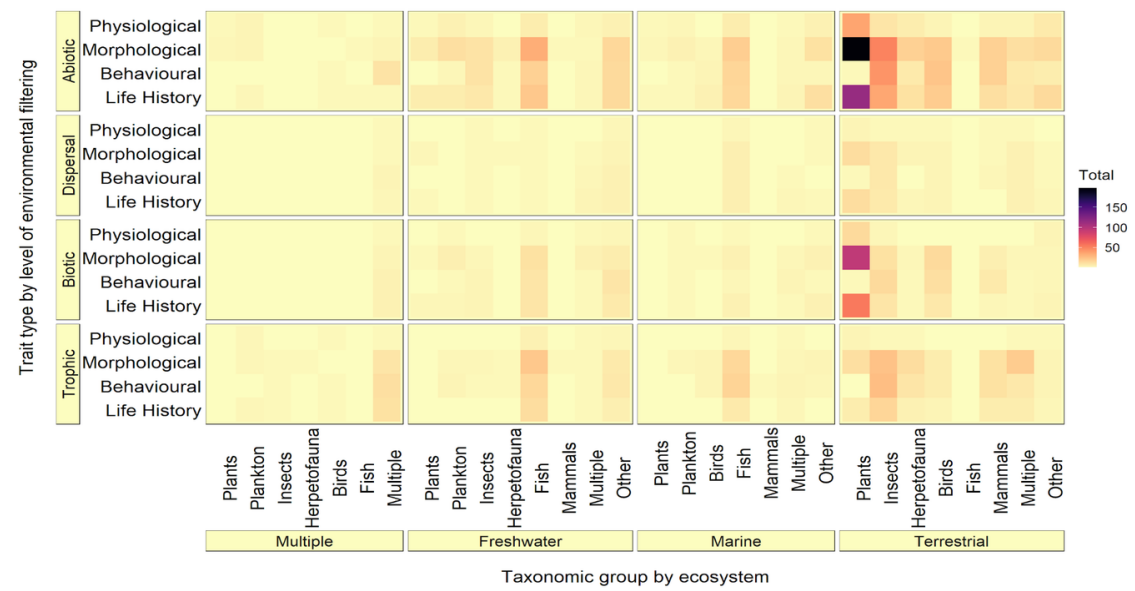

\section{Figure 3}

Figure 3. Visualizing trait-based ecology research by ecosystem type and taxonomic focus (x-axis), and level of environmental filtering and trait type (y-axis). Colour intensity relates to the number of research studies at the nexus of these domains.

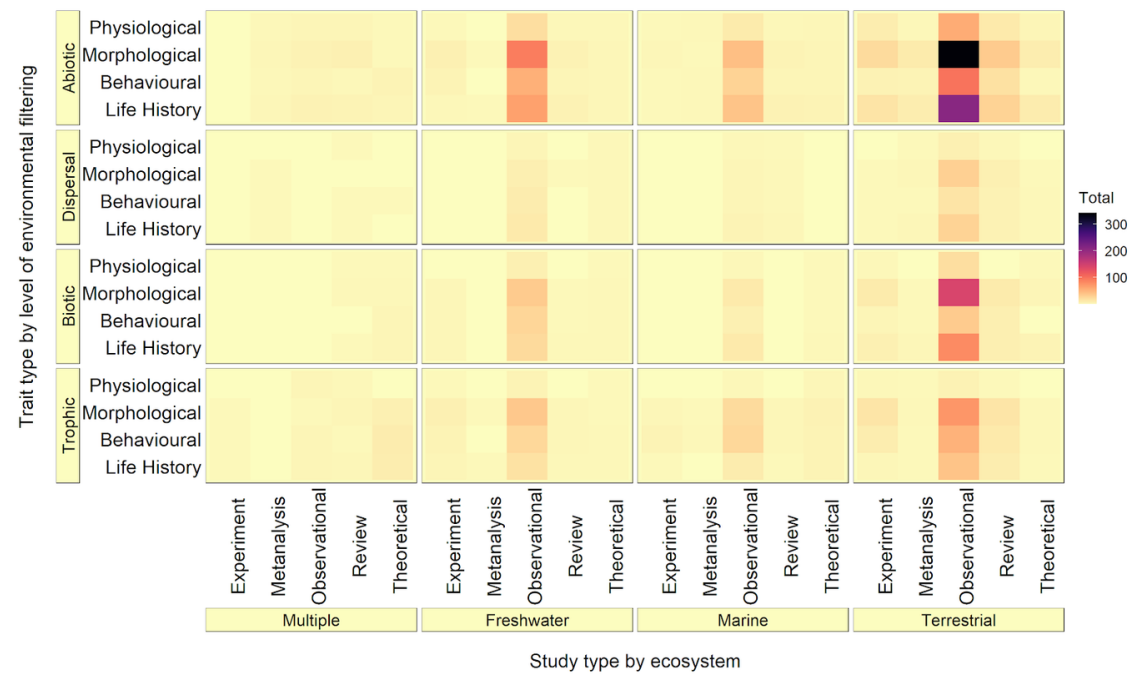

\section{Figure 4}

Figure 4. Visualizing trait-based ecology research by ecosystem and type of study (x-axis), and level of environmental filtering and trait type (y-axis). Colour intensity relates to the number of research studies at the nexus of these domains. 


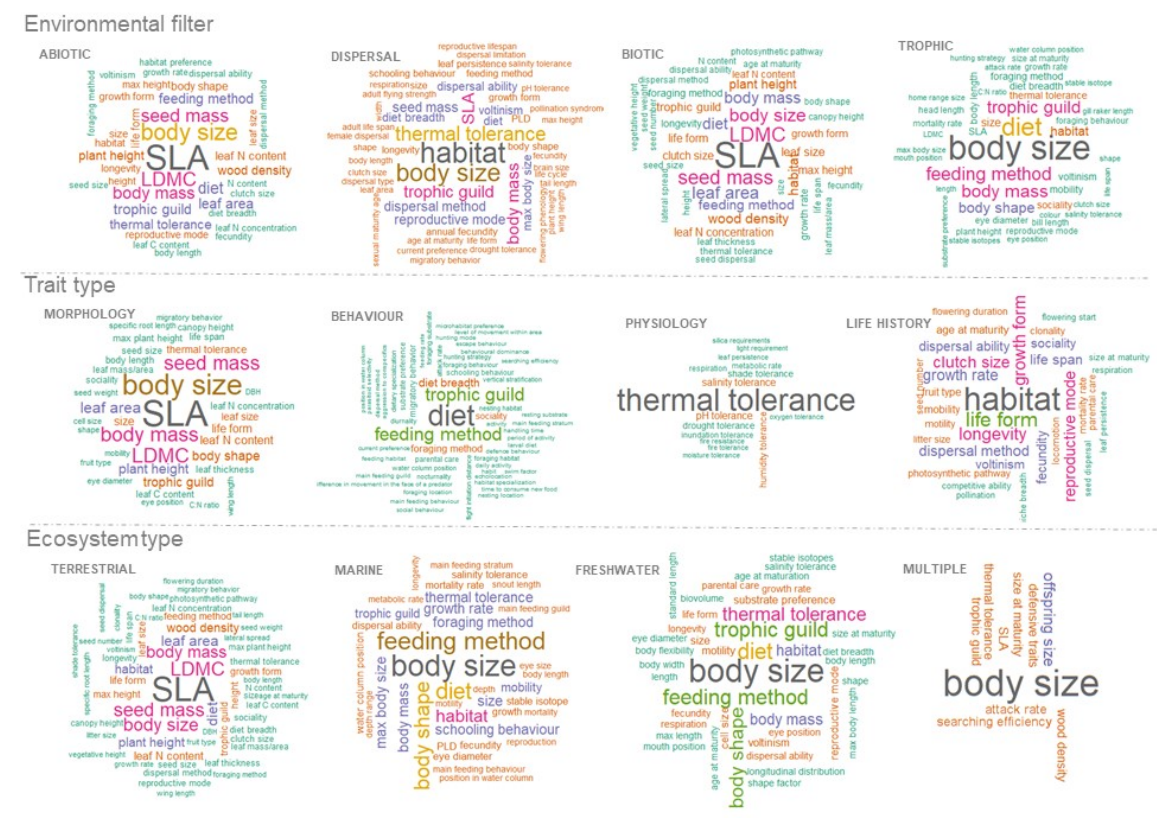

Figure 5

Figure 5. Top traits within ecological research on environmental filtering of species distribution and abundance. Word size is proportional to the relative number of studies focused on each trait by environmental filtering process. SLA = specific leaf area; PLD = pelagic larval duration; LDMC = leaf dry matter content. Note that this plot is visualized on the level of the individual study, and multiple filters/trait types could be used by a single study. 


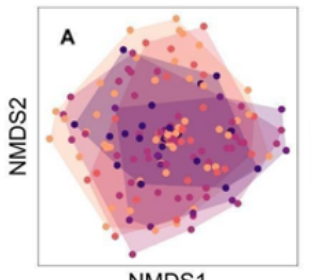

Taxonomic group

- Vertebrate

- Invertebrate

- Plants

- Plants

- Multiple

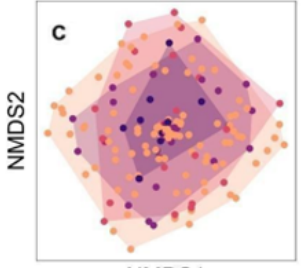

Ecosystem

- Terrestrial

- Marine

- Freshwate

- Broad

NMDS1

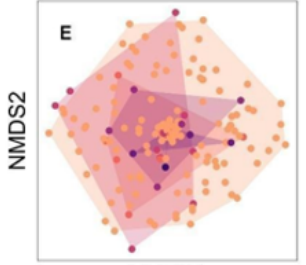

NMDS1

- Exploitation

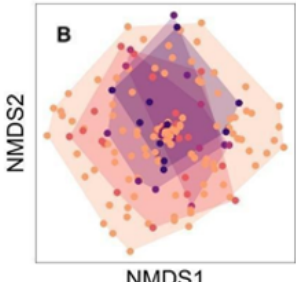

Study type

Observationa

Experiment

Review

- Theory

NMDS1

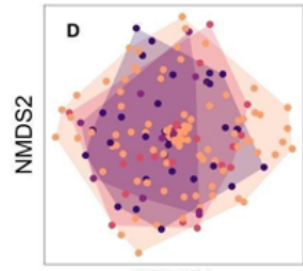

Filte

- Abiotic

- Biotic

- Dispersal

NMDS1

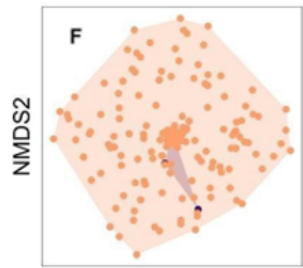

Predictive

- Yes

NMDS1

\section{Figure 6}

Figure 6. Non-metric multidimensional scaling (nMDS) plot visualizing the multivariate assemblage of traits applied within ecological research (each point = one study) according to: A) driver(s) of global change examined, B) level of environmental filtering, C) whether or not the studies generated ecological predictions, D) the type of study, E) focal ecosystem, and F) focal taxonomic group. Two-dimensional distance between points represents dissimilarity between multivariate trait sets in each study; shaded area represents the multivariate space occupied by studies in each level of the corresponding grouping factor. 


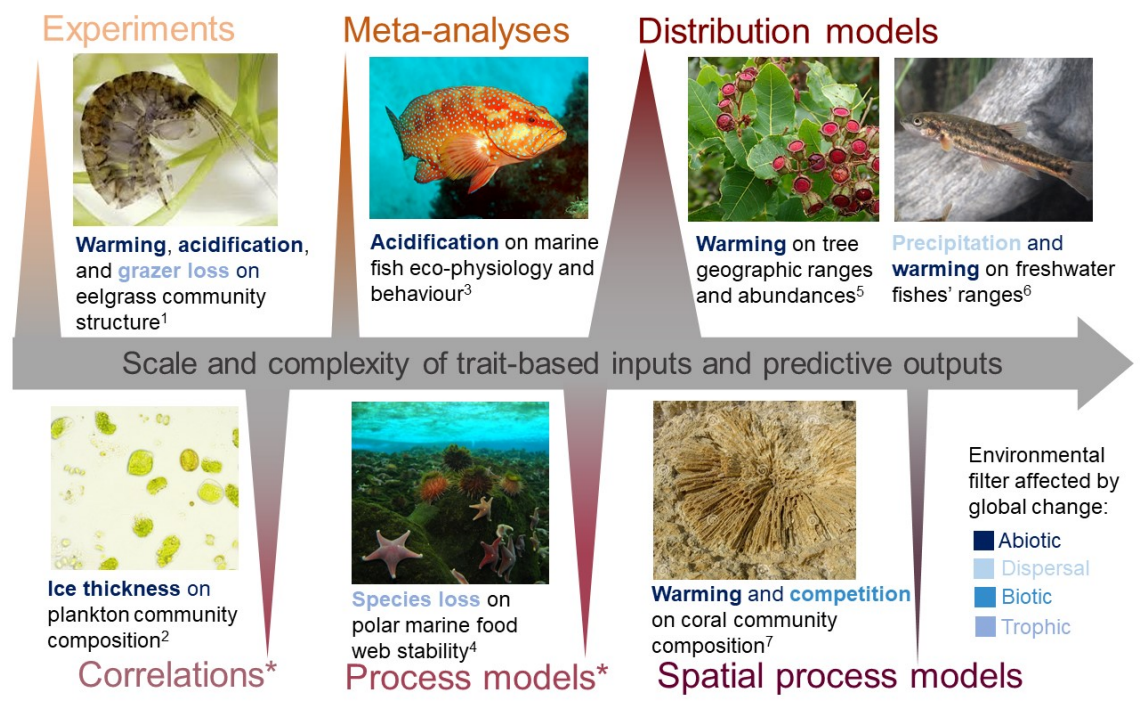

Figure 7

Figure 7. A variety of methods generate traits-based predictions of ecological response to global change. Approaches vary in the scale and resolution of trait data inputs and predictive outputs (see Fig 1). To date, trait-based studies focused on global change prediction have been limited ( $<3 \%$ of 822 papers). Here we highlight some of the studies that explicitly predict future ecological outcomes based on trait-based interactions, and types of environmental filtering they consider. Size of the grey triangle represents the relative abundance of each study type within 22 predictive global change papers; trait-based distribution models are most common, while spatially explicit trait-based process models are least common. * Analyses are not spatially explicit. ${ }^{1}$ Eckloff et al. 2015, ${ }^{2}$ Ozkundakci et al. 2016, ${ }^{3}$ Cattano et al. $2018,{ }^{4}$ Jacob et al. 2011, ${ }^{5}$ Fordham et al. 2012, ${ }^{6}$ Whitney et al. 2017, ${ }^{7}$ Edmunds et al. 2014. 


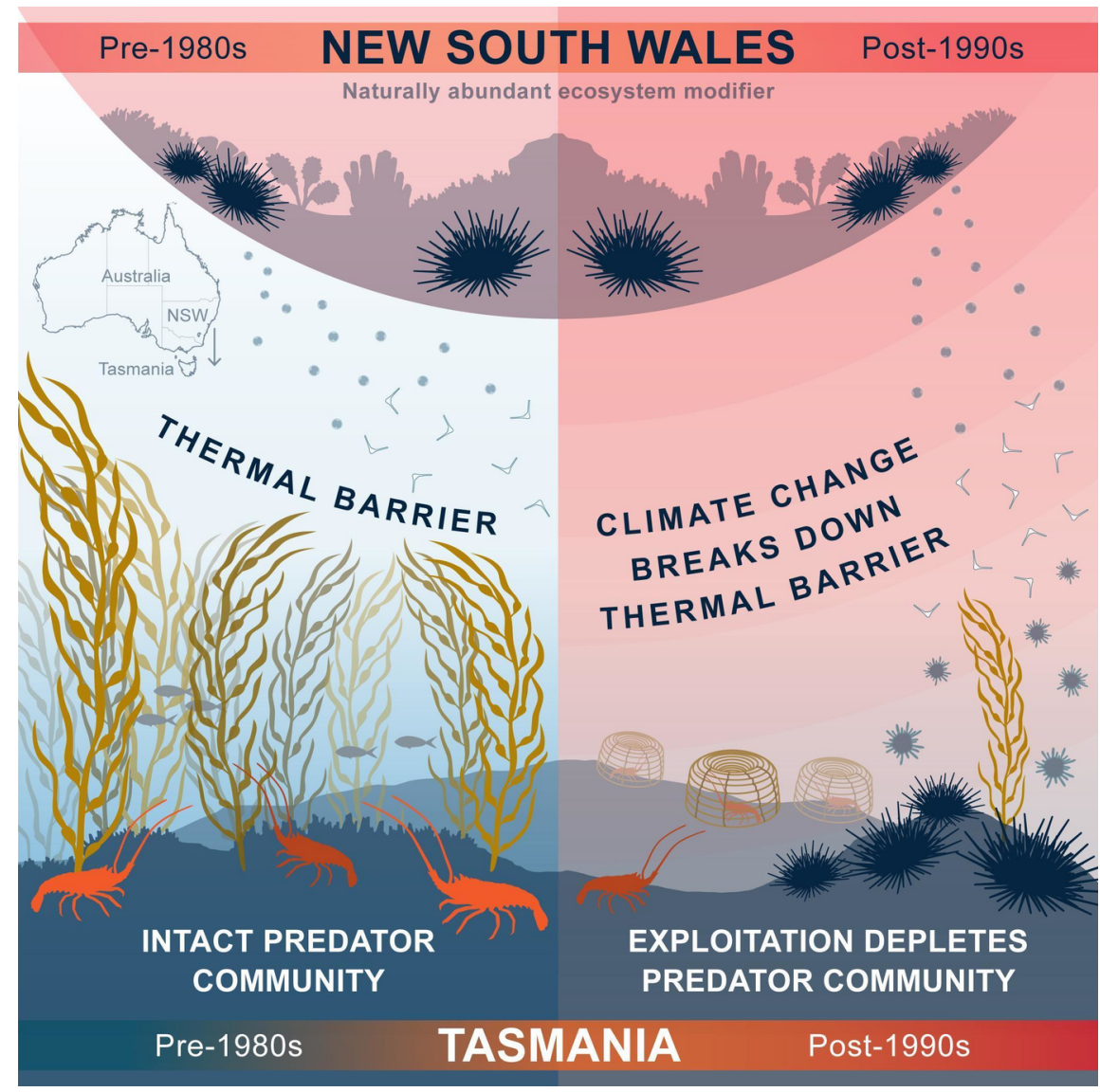

\section{Figure 8}

Figure 8. Can trait-based environmental filtering explain ecosystem shifts under global change? By the late 1990s, climate change and over-exploitation had both begun to alter environmental filters on marine species' abiotic, dispersal, and biotic traits, leading to rapid range expansion of the ecosystem engineer Centrlostephanus rodgersii from New South Wales (NSW) into Tasmania in Southeastern Australia. Ocean warming raised coastal temperatures off Tasmania above critical thermal limits for $C$. rodgersii larval survival (a key abiotic trait). A long larval phase and resistance to starvation (two dispersal traits) facilitated C. rodgersii colonization of Tasmania from NSW, where populations of nocturnal predators adapted to crushing bottom-dwelling prey such as C. rodgersii (trophic interaction traits) had been depleted due to over-harvesting. 